\title{
Environmental stress impairs photoreceptor outer segment (POS) phagocytosis and degradation and induces autofluorescent material accumulation in hiPSC-RPE cells
}

Sonal Dalvi 1,2, Chad A. Galloway ${ }^{1,2,5}$, Lauren Winschel ${ }^{1,2}$, Ali Hashim,2, Celia Soto ${ }^{1,2}$, Cynthia Tang ${ }^{1,2}$, Leslie A. MacDonald ${ }^{1,2}$ and Ruchira Singh ${ }^{1,2,3,4}$

\begin{abstract}
Retinal pigment epithelium (RPE) cell dysfunction is central to the pathogenesis of age-related macular degeneration (AMD), a leading cause of adult blindness. Aging, the single biggest risk factor for AMD development, favors increase in RPE autofluorescent material due to accumulation of POS-digestion by-products through lysosomal dysfunction and impaired POS degradation. Apart from aging, environmental agents affect lysosomal function in multiple model systems and are implicated in AMD. Iron (Fe) overload and cigarette smoke exposure are the two environmental factors that are known to affect the lysosomal pathway and impact RPE cell health. However, the impact of Fe and cigarette smoke, on POS processing and its consequence for autofluorescent material accumulation in human RPE cells are yet to be established. Human induced pluripotent stem cell (hiPSC)-derived RPE, which phagocytoses and degrades POS in culture and can be derived from control individuals (no history/susceptibility for retinal disease), provides a model system to investigate the singular effect of excess Fe and/or cigarette smoke on POS processing by RPE cells. Using at least three distinct control hiPSC lines, we show that, compared to untreated hiPSC-RPE cells, POS uptake is reduced in both Fe (ferric ammonium citrate or FAC) and FAC + CSE (cigarette smoke extract)-treated hiPSCRPE cells. Furthermore, exposure of hiPSC-RPE cultures to FAC + CSE leads to reduced levels of active cathepsin-D (CTSD), a lysosomal enzyme involved in POS processing, and causes delayed degradation of POS. Notably, delayed degradation of POS over time (2 weeks) in hiPSC-RPE cells exposed to Fe and CSE was sufficient to increase autofluorescent material build-up in these cells. Given that inefficient POS processing-mediated autofluorescent material accumulation in RPE cells has already been linked to AMD development, our results implicate a causative role of environmental agents, like Fe and cigarette smoke, in AMD.
\end{abstract}

Correspondence: Ruchira Singh (ruchira_singh@urmc.rochester.edu) 'Department of Ophthalmology (Flaum Eye Institute), University of Rochester, Rochester, NY, USA

${ }^{2}$ Department of Biomedical Genetics, University of Rochester, Rochester, NY, USA

Full list of author information is available at the end of the article.

Edited by A. Rufini

\section{Introduction}

Clinical and basic science research has revealed a significant role of environmental agents in the etiology of macular degeneration (MD). In particular, twin studies have shown that inheritance of MD ranges from $45 \%$ for early maculopathy to $71 \%$ for advanced agerelated macular degeneration (AMD), supporting a genetic component but also emphasizing the role of

\section{(c) The Author(s) 2019}

(c) (i) Open Access This article is licensed under a Creative Commons Attribution 4.0 International License, which permits use, sharing, adaptation, distribution and reproduction cc. in any medium or format, as long as you give appropriate credit to the original author(s) and the source, provide a link to the Creative Commons license, and indicate if changes were made. The images or other third party material in this article are included in the article's Creative Commons license, unless indicated otherwise in a credit line to the material. If material is not included in the article's Creative Commons license and your intended use is not permitted by statutory regulation or exceeds the permitted use, you will need to obtain permission directly from the copyright holder. To view a copy of this license, visit http://creativecommons.org/licenses/by/4.0/. 
environmental factors ${ }^{1}$. Two prominent environmental agents linked to AMD through epidemiological and experimental studies are excess iron $(\mathrm{Fe})$ and cigarette smoke $^{2-8}$. Interestingly, aging, a necessary requirement for AMD development, has been associated with increased Fe levels in both the mouse and human/ retina ${ }^{6,9,10}$. Furthermore, cadaver eyes from patients with AMD show increased Fe deposits in retinal pigment epithelium (RPE) cells ${ }^{11}$. Importantly, mutations in CP gene lead to increased Fe in RPE cells/retina and cause maculopathy-like features in patients with aceruloplasminemia ${ }^{12,13}$. In addition, targeting Fe homeostasis through genetic ablation in rodent models has been shown to cause local Fe accumulation within RPE cells and maculopathy-relevant cellular changes ${ }^{14-16}$. Similarly, exposure risk for cigarette smoke, a promi-

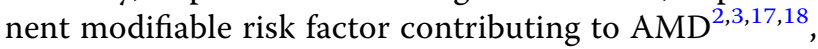
is higher in adults aged $18-64^{19}$. Furthermore, chronic exposure to cigarette smoke in mice results in pathological alterations consistent with $\mathrm{AMD}^{4}$. Likewise, acute exposure of ARPE-19 cells, primary human RPE, and human fetal RPE to cigarette smoke extract (CSE) and/or toxic components of cigarette smoke such as [B (a)P] and acrolein leads to cellular alterations consistent with AMD (e.g., oxidative stress, increased autophagy, and cell death $)^{5,20,21}$.

The accumulation of autofluorescent material (lipofuscin), metabolic debris from incomplete photoreceptor outer segment (POS) digestion, has been linked to AMD development through several plausible mechanisms, reduction in RPE cytoplasmic volume ${ }^{22}$, complement activation $^{23}$, and RPE cell death ${ }^{24}$. In fact, aging, the biggest risk factor for AMD development, leads to a significant increase in RPE lipofuscin accumulation, with $\sim 1 \%$ of the RPE cytoplasmic volume covered by lipofuscin in the first decade of life compared to $\sim 19 \%$ by the age $80^{25}$. Interestingly, increased autofluorescent material accumulation in the RPE cells and RPE Fe overload have been reported to coexist in patients with aceruloplasminemia ${ }^{12,26}$. Furthermore, excess Fe in cells has been shown to selectively accumulate in lysosomes as a component of Fe-rich lipofuscin ${ }^{27,28}$. In fact, in ARPE-19 cells, excess Fe has been shown to alter the activity of cathepsin-D (CTSD $)^{6}$, a lysosomal enzyme involved in degradation of $\operatorname{POS}^{29,30}$. Similarly, cigarette smoke has been linked to lysosomal dysfunction ${ }^{31,32}$ and altered CTSD activity in ARPE-19 cells and a murine model exposed to $[\mathrm{B}(\mathrm{a}) \mathrm{P}]^{32}$. Although these data indicate that like aging, cigarette smoke and Fe can influence POS processing, the impact of $\mathrm{Fe}$ and cigarette smoke on POS phagocytosis and degradation, and its consequence for accumulation of autofluorescent POS-digestion by-products, a pathological feature of AMD, have not been established in human RPE cells.
Human induced pluripotent stem cell (hiPSC) technology has provided a suitable platform to gain fundamental insights into several RPE-based disorders, including AMD and related MDs. For instance, hiPSCRPE derived from patients with AMD and related macular dystrophies, Sorsby's fundus dystrophy (SFD) and Doyne honeycomb retinal dystrophy, have shown the ability to mimic both disease-associated molecular alterations with complement pathway alteration ${ }^{33,34}$ and pathological changes such as drusen formation and extracellular matrix protein accumulation ${ }^{34,35}$. Notably, disease modeling efforts using hiPSC-RPE-derived cell models have utilized the unique ability to select a specific patient population to investigate the (i) precise impact of genetic defects on monogenic diseases with complete penetrance [e.g., best disease (BD) ${ }^{36,37}, \mathrm{SFD}^{34}$, and MERTK mutations in Retinitis pigmentosa (RP) $)^{38,39}$, as well as the (ii) consequence of a specific protective/risk haplotype in individual genes (e.g., $C F H^{35}$ ) in a complex multifactorial disease like AMD. Although genetic defects have been central to disease modeling efforts using hiPSC-RPE cells, it is plausible that hiPSC-RPE from healthy individuals with no history of retinal disease provides a suitable platform to interrogate the singular role of environmental agents in the development of disease-relevant molecular and pathological changes in human RPE cells. This is especially pertinent to cellular process(es), such as phagocytosis and degradation of POS, and linked pathological changes, such as increased accumulation of autofluorescent material, that can be faithfully mimicked in an hiPSC-RPE model system.

In this study, we utilized hiPSC-RPE from at least three distinct control hiPSC lines, with no known genetic susceptibility for AMD development, to evaluate the singular impact of cigarette smoke (CSE, $0.5 \% /$ day) and/or Fe (ferric ammonium citrate or FAC, 50 and $200 \mu \mathrm{g} / \mathrm{ml} /$ day) on (i) lysosomal function and POS processing and (ii) its consequence for autofluorescent material accumulation in RPE cells.

\section{Results}

\section{Control hiPSC-RPE lines used in this study display key morphological, protein expression, and functional attributes of RPE cell in vivo}

Electron microscopy analyses of hiPSC-RPE lines utilized in this study consistently displayed typical RPE features, including microvilli and apically migrating melanosomes (Fig. 1a). Furthermore, transepithelial resistance (TER) measurements of hiPSC-RPE cultures derived from distinct hiPSC lines showed the presence of functional tight junctions with (TER) $\geq 150 \Omega \mathrm{cm}^{2}$, an in vivo reported threshold of $\mathrm{TER}^{40}$ (Fig. 1b). Immunocytochemical analyses of hiPSC-RPE lines further demonstrated expected pattern and robust expression of 


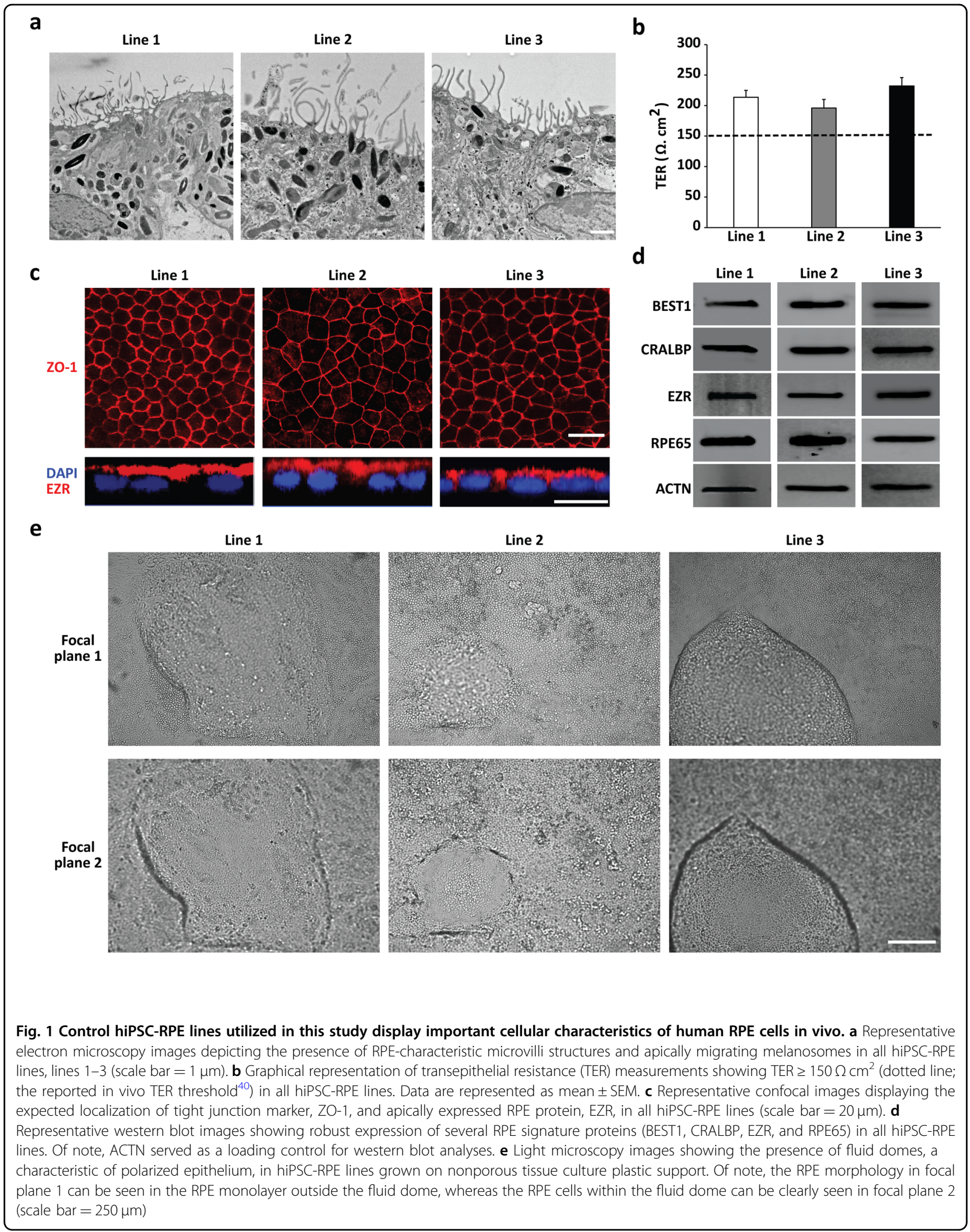


tight junction marker, ZO-1, (Fig. 1c) and apically localized RPE protein, EZR (Fig. 1c). Additionally, western blot analyses revealed strong and similar expression of several RPE signature proteins, BEST1, CRALBP, EZR, and RPE65, in the hiPSC-RPE lines (Fig. 1d). Of note, ACTN served as a loading control for western blot analyses experiments (Fig. 1d). Lastly, consistent with the formation of a polarized RPE monolayer, when hiPSCRPE cell lines were grown on nonpermeable plastic support, they displayed the presence of fluid domes ${ }^{34,36}$ (Fig. 1e).

\section{Acute exposure to excess $\mathrm{Fe}(\mathrm{FAC})$ is sufficient to impair POS phagocytosis by hiPSC-RPE cells}

Given that the amount of FAC $(50-200 \mu \mathrm{g} / \mathrm{ml})^{41-44}$ utilized in this study to assess the acute $(24 \mathrm{~h})$ and chronic ( 2 weeks to 1 month) impact of excess Fe was based on previously published studies in primary RPE cell cultures (mouse and human fetal RPE) and immortalized cell lines (HepG2 and ARPE-19), we first confirmed that FAC exposure was sufficient to elicit the expected (i) intracellular accumulation of Fe deposits (Fig. 2a) and (ii) altered expression of Fe-regulatory genes (CP, HFE, GSS, and TF) (Fig. 2b, Supplementary Table 1) in FAC-treated hiPSCRPE cultures. Next, to determine the impact of excess Fe on POS phagocytosis and degradation, hiPSC-RPE cells were simultaneously fed POS (20 POS/RPE cell) and exposed to FAC (Fig. 2c). POS uptake (2 $\mathrm{h}$ post-POS feeding; $0 \mathrm{~h}$ time point) and degradation ( $24 \mathrm{~h}$ post-POS feeding; $24 \mathrm{~h}$ time point) were then determined by quantification of RHO, a POS-specific protein (Fig. 2c). With regard to RHO quantification, the cumulative amount of RHO, including monomer, dimer, and multimers ${ }^{36}$ and potentially the post-translationally modified forms (e.g., glycosylated RHO $)^{45}$ relative to loading control ACTN, was utilized to assess the amount of RHO/ POS in hiPSC-RPE cultures. Of note, parallel cultures of hiPSC-RPE fed with only POS served as untreated controls in these experiments. Consistent with a prior study in the ARPE-19 cell line that showed reduced POS phagocytosis in the presence of excess $\mathrm{Fe}^{6}$, quantitative western blot analyses of FAC-treated versus untreated hiPSC-RPE cultures revealed lower levels of RHO/POS in the FAC-treated hiPSC-RPE cells $(22.82 \pm 9.42 \%)$ at the $0 \mathrm{~h}$ time point compared to the untreated hiPSC-RPE cells $(100 \pm 49.37 \%, P \leq 0.01)$ (Fig. $2 d$, e). In contrast, no difference in the rate of RHO/POS degradation, the amount of ingested POS degraded within $24 \mathrm{~h}$, was observed between untreated $(51.42 \pm 7.96 \%)$ and FAC-treated $(37.68 \pm 15.49 \%)$ hiPSC-RPE cultures (Fig. 2d, f). Notably, acute exposure to FAC did not adversely impact cell viability (Fig. 2g, top panel) or morphology (Supplementary Fig. 1) of hiPSC-RPE cultures. Furthermore, similar localization of tight junction marker, ZO-1 (Fig. 2g, bottom panel), and TER measurements (Fig. 2h) were seen in untreated versus FAC-treated hiPSC-RPE cultures. Altogether, these results showed that FAC supplementation of cell culture media can alter Fe homeostasis and cause Fe overload in hiPSC-RPE cultures. Furthermore, nontoxic levels of excess Fe by itself in the absence of a specific gene defect is sufficient to impair POS uptake in hiPSC-RPE cells.

\section{Acute exposure to cigarette smoke extract (CSE) has no effect on POS phagocytosis and degradation by hiPSC-RPE cells}

CSE at a concentration of $0.5 \%$, a dose that has previously been shown to affect ARPE-19 cell health and function $^{5}$, was used to assess the effect of cigarette smoke on POS phagocytosis and degradation by hiPSC-RPE cells. Importantly, consistent with previously described regulation of MMP-1 activity by cigarette smoke ${ }^{46}$, chronic exposure (2 weeks) of hiPSC-RPE cultures to CSE led to increased activity of secreted MMP-1 (Fig. 3a). Furthermore, hiPSC-RPE cultures supplemented with CSE $(0.5 \%)^{5}$ showed constant levels of CSE, as measured by absorbance at $320 \mathrm{~nm}$, for the $24 \mathrm{~h}$ period of daily CSE treatment (Fig. 3b). To evaluate the effect of cigarette smoke on POS phagocytosis and degradation by hiPSCRPE cells, we utilized acute (24h) CSE exposure as the extrinsic stressor in the previously described phagocytosis and degradation experiment (Fig. 2c). In contrast to FACtreated RPE cells (Fig. 2d-f), western blot analyses of untreated versus CSE-treated hiPSC-RPE cells showed no difference in $\mathrm{RHO} / \mathrm{POS}$ abundance at the $0 \mathrm{~h}$ time point (CSE-treated: $104.21 \pm 75.8 \%$ versus untreated: $100 \pm$ $59.78 \%, P=0.55$ ) (Fig. 3c, d). Furthermore, untreated and CSE-treated hiPSC-RPE cells degraded a similar amount of $\mathrm{RHO}$ at the $24 \mathrm{~h}$ time point (CSE-treated: $44.62 \pm$ $10.26 \%$ versus untreated: $53.17 \pm 3.94 \%, P=0.51$ ) (Fig. 3c, e). In addition, CSE exposure $(24 \mathrm{~h})$ did not impact the cell viability (Fig. 3f, top panel), morphology (Supplementary Fig. 1), ZO-1 localization (Fig. 3f, bottom panel), and TER (Fig. 3g) in hiPSC-RPE cultures. Taken together, these results show that although supplementation of hiPSC-RPE cultures with a nontoxic dose of CSE can elicit the documented smoke-induced change in MMP-1 activity $^{46}$, CSE exposure by itself is not sufficient to adversely affect POS phagocytosis and degradation.

\section{Combined exposure to FAC and CSE affects active-CTSD levels and impairs both POS phagocytosis and degradation in hiPSC-RPE cells}

Although several studies have evaluated the impact of Fe or cigarette smoke exposure on RPE cell health in vitro and in vivo ${ }^{4-6,14,16,47}$, the impact of combined exposure on Fe and CSE together has not been evaluated. Simultaneous presence of iron overload in RPE cells and 


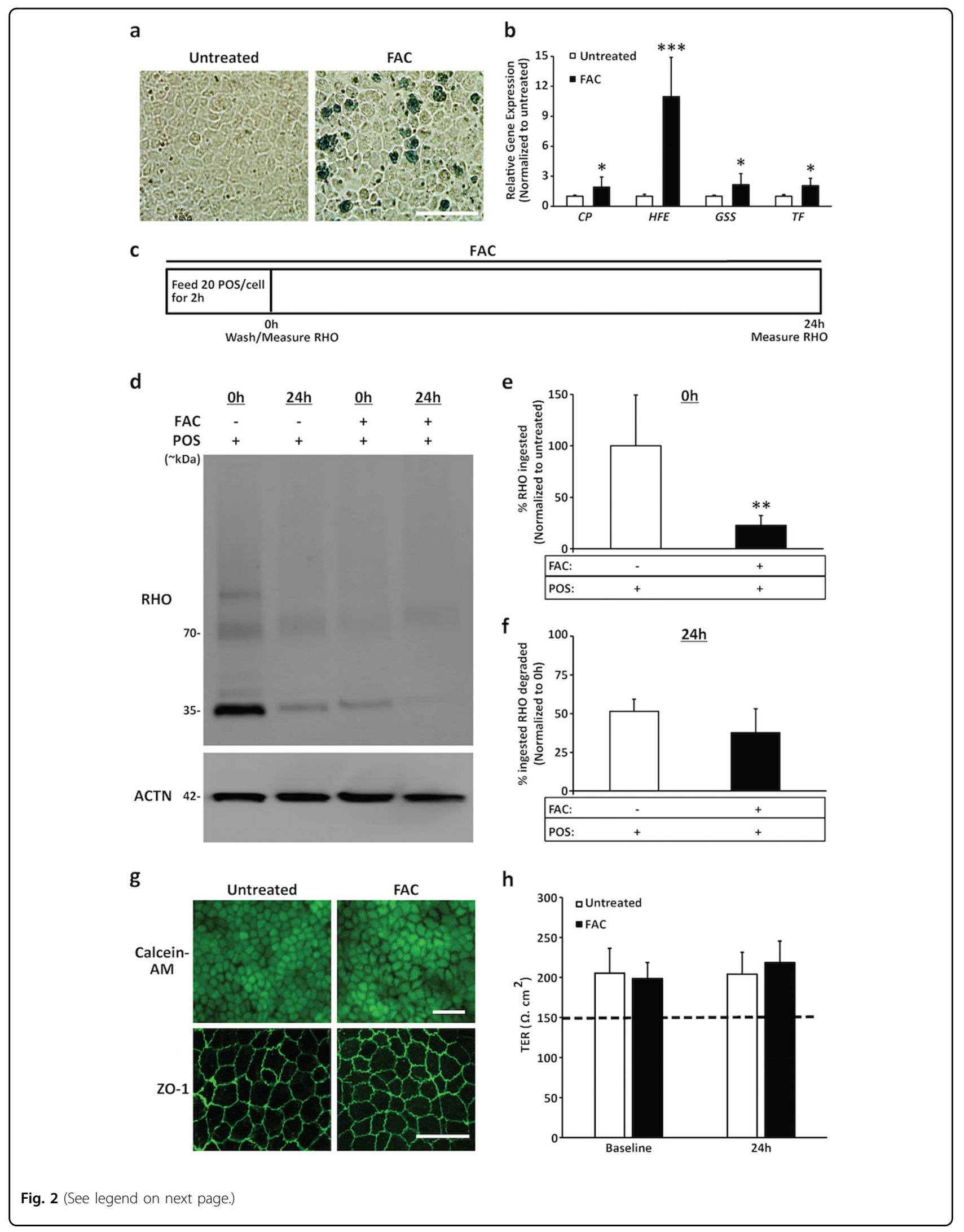


(see figure on previous page)

Fig. 2 FAC supplementation of culture media is sufficient to alter iron ( $\mathrm{Fe})$ metabolism and impair POS uptake by hiPSC-RPE cells. a Representative images of parallel cultures of Prussian blue-stained untreated and FAC-treated $(50 \mu \mathrm{g} / \mathrm{ml}, \sim 1 \mathrm{month})$ hiPSC-RPE cells showing increased presence of Fe deposits in FAC-treated hiPSC-RPE cells (scale bar $=50 \mu \mathrm{m}$ ). b Quantitative analyses of real-time PCR demonstrating increased expression of iron-regulatory genes (CP, HFE, GSS, and TF) in untreated versus FAC-treated $(50 \mu \mathrm{g} / \mathrm{ml}, \sim 1$ month) hiPSC-RPE cultures. Data are represented as mean \pm SEM. ${ }^{*} P \leq 0.05,{ }^{* * *} P \leq 0.001, n=3$ independent trials. c A schematic depicting the protocol utilized for assessing the impact of acute FAC exposure $(200 \mathrm{\mu g} / \mathrm{ml}, 24 \mathrm{~h}$ ) on POS uptake ( $0 \mathrm{~h}$ time point) and degradation ( $24 \mathrm{~h}$ time point) post $2 \mathrm{~h} \mathrm{POS} \mathrm{feeding} \mathrm{(20} \mathrm{POS/RPE}$ $\left(\right.$ cell) $^{36}$. Of note, the amount of POS in hiPSC-RPE cells was evaluated indirectly by measuring the protein levels of RHO, a POS-specific protein. $\mathbf{d}-\mathbf{f}$ Representative western blot images (d) and corresponding quantitative analyses (e, $\mathbf{f})$ showing reduced uptake of RHO/POS at the $0 \mathrm{~h}$ time point $\mathbf{( d )}$ e) but similar degradation of ingested RHO at the $24 \mathrm{~h}$ time point $(\mathbf{d}, \mathbf{f})$ in untreated versus FAC-treated hiPSC-RPE cultures. Data are presented as mean \pm SEM. ${ }^{* *} P \leq 0.01, n=3$ independent trials. Of note, to represent the rate of POS degradation, the quantification of RHO levels at $24 \mathrm{~h}$ time point is calculated relative to the amount of POS uptake ( $0 \mathrm{~h}$ time point). Note: ACTN served as loading control for western blot analyses. $\mathbf{g}$ Representative fluorescent microscopy (top panel) and confocal microscopy images (bottom panel) showing no difference in cell viability (calceinAM) and localization of tight junction marker, ZO-1, in untreated versus FAC-treated ( $200 \mu \mathrm{g} / \mathrm{ml}, 24 \mathrm{~h}$ ) hiPSC-RPE cultures (scale bar $=20 \mu \mathrm{m})$. $\mathbf{h}$ Quantitative analyses of TER measurements showing similar TER values with TER $\geq 150 \Omega \mathrm{cm}^{2}$ (dotted line; the reported in vivo TER threshold ${ }^{40}$ ) at baseline and the $24 \mathrm{~h}$ time point in both untreated and FAC-treated $(200 \mu \mathrm{g} / \mathrm{ml}, 24 \mathrm{~h})$ hiPSC-RPE cultures. Data are presented as mean \pm SEM. $n=3$ independent trials

cigarette smoke exposure is a plausible scenario given the fact that aging has been shown to increase the levels of Fe in RPE cells ${ }^{9}$ and cigarette smoke is a significant risk factor linked to adult population ${ }^{19}$. Furthermore, given that lifestyle and dietary habits have been implicated in both smoke exposure (smoking cigarettes) ${ }^{2}$ and iron overload (eating red meat ${ }^{48}$ ) in AMD, it is not unlikely that excess $\mathrm{Fe}$ and cigarette smoke exposure together occur in a subset of people in the general population. Additionally, RPE iron overload and cigarette smoke exposure can occur together in patients with hemochromatosis who have iron overload in RPE cells ${ }^{12}$.

Consistent with the reduced POS uptake in FAC-treated cultures (Fig. 2d, e), FAC + CSE-treated hiPSC-RPE cells showed decreased amount of RHO at the $0 \mathrm{~h}$ time point when compared to untreated hiPSC-RPE cells (FAC + CSE-treated: $44.92 \pm 22.04 \%$ versus untreated: $100 \pm$ $22.41 \%, P \leq 0.05$, Fig. 4a, b). Furthermore, combined FAC and CSE treatment led to a decreased rate of $\mathrm{RHO} / \mathrm{POS}$ degradation at the $24 \mathrm{~h}$ time point (FAC + CSE-treated: $19.46 \pm 8.54 \%$ versus untreated: $65.49 \pm 8.39 \%, P \leq 0.05$, Fig. 4a, c). A plausible role of impaired lysosomal function in decreased rate of POS degradation observed in FAC + CSE-treated cultures, was implied by the negative impact of FAC + CSE treatment on active-CTSD levels (Fig. 4d, e). Of note, CTSD is an integral lysosomal enzyme involved in POS degradation ${ }^{29,30,49}$. Specifically, slower POS degradation in FAC + CSE-treated cultures correlated with reduced active-CTSD levels (FAC + CSE-treated: $0.54 \pm 0.08$ versus untreated: $1 \pm 0.23, P \leq 0.001$, Fig. $4 \mathrm{~d}, \mathrm{e})$. In contrast, no change in the expression of proCTSD was seen between untreated and FAC + CSEtreated hiPSC-RPE cultures (FAC + CSE-treated: $0.98 \pm$ 0.41 versus untreated: $1 \pm 35, P=0.76$, Supplementary Fig. 2). To further confirm the correlation of reduced active-CTSD abundance with decreased POS degradation, we also determined the effect of FAC supplementation, a stressor that reduced POS uptake but not degradation, on active-CTSD levels in hiPSC-RPE cultures. Consistent with similar rate of POS digestion (Fig. 2d, f), untreated versus FAC-treated cultures showed similar expression of active-CTSD (FAC-treated: $0.96 \pm 0.11$ versus untreated: $1 \pm 0.18, P=0.76$ ) (Supplementary Fig. 3). Notably, similar to FAC and CSE treatment, acute exposure $(24 \mathrm{~h})$ of hiPSC-RPE to FAC + CSE had no adverse effects on cell viability (Fig. 4f, top panel), RPE morphology (Supplementary Fig. 1), ZO-1 localization (Fig. 4f, bottom panel), and TER values (untreated: $229.13 \pm 41.9, F A C+$ CSE treated: $237.16 \pm 34.86, P=0.58$ ) (Fig. $4 \mathrm{~g}$ ). Altogether, these results show that combined exposure to excess Fe and cigarette smoke is sufficient to reduce the levels of active-CTSD, a POS processing lysosomal enzyme, and negatively impact POS degradation by hiPSC-RPE cells.

\section{Chronic exposure to FAC and CSE is sufficient to induce increased autofluorescent material accumulation in hiPSC- RPE cells}

Our previous results had shown that addition of FAC and/or CSE to the cell culture media for short duration (24 h) does not adversely impact hiPSC-RPE morphology, TER and cell viability (Figs. 2-4 and Supplementary Fig. 1). However, before evaluating the impact of FAC + CSE treatment on autofluorescent material accumulation after daily POS feeding for 2 weeks, we wanted to ensure that simultaneous stress of daily POS feeding and FAC + CSE exposure together for chronic duration (2 weeks) was not toxic to the cells. In these experiments, parallel cultures of hiPSC-RPE cells were either designated as (i) "untreated cultures" and fed daily with only a physiologic dose of POS ( 20 POS per RPE cell per day) or (ii) "FAC + CSEtreated cultures" and were simultaneously exposed to both POS and FAC + CSE on a daily basis. Notably, collective stress of chronic POS feeding in conjunction with FAC and CSE exposure, was not detrimental to hiPSC- 
a

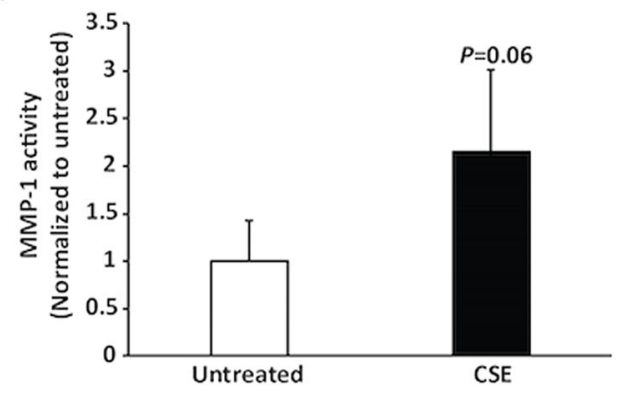

C

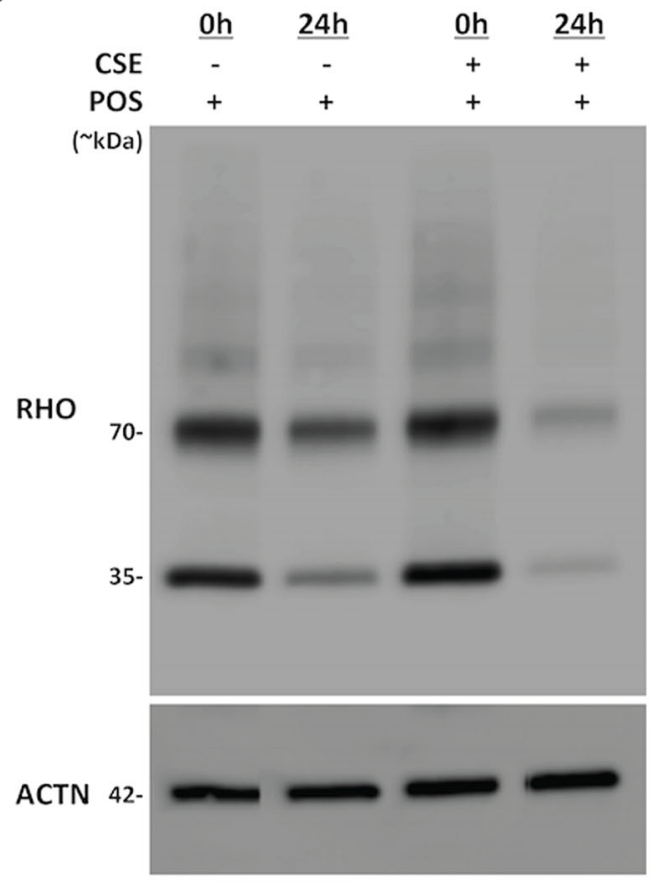

f
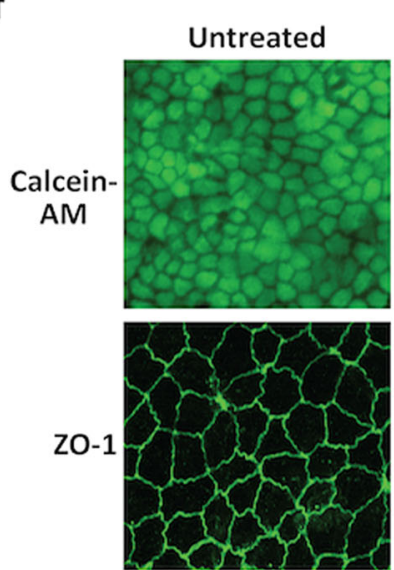

b

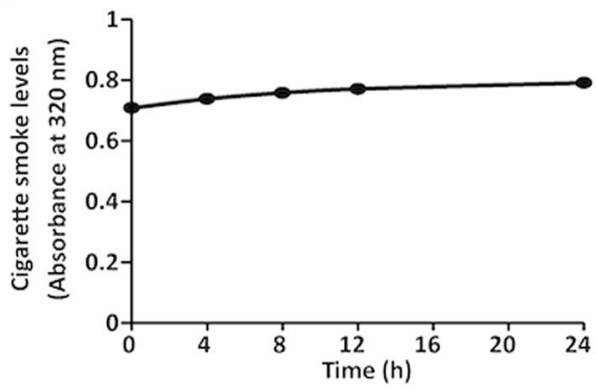

d

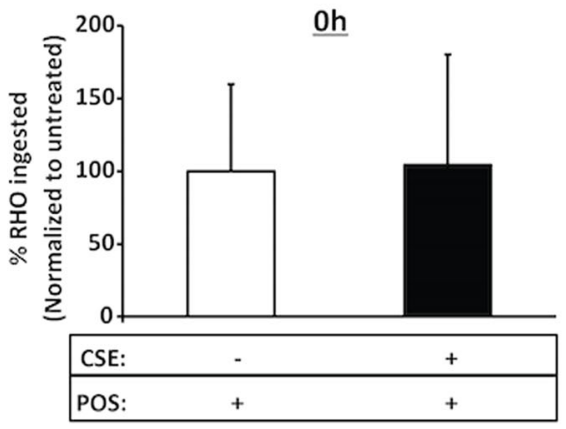

e

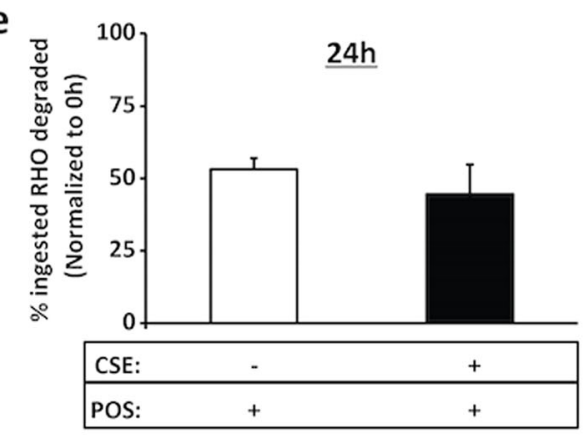

g

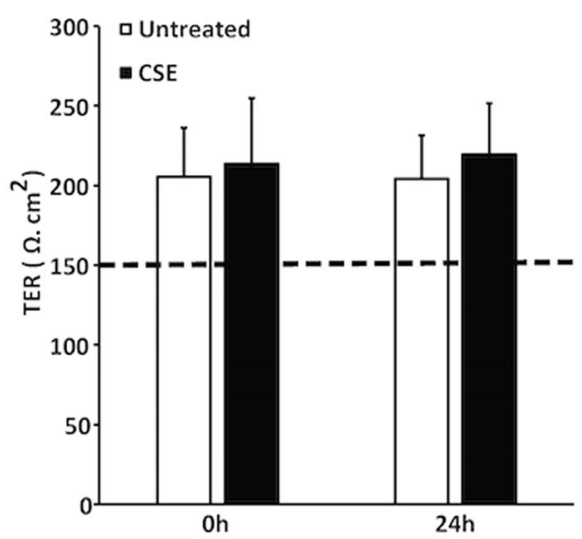

Fig. 3 (See legend on next page.) 
(see figure on previous page)

Fig. 3 CSE supplementation of culture media is sufficient to alter MMP-1 activity but has no impact on POS phagocytosis and degradation by hiPSC-RPE cells. a Zymography analyses of basally secreted media from parallel cultures of untreated versus CSE-treated (0.5\%, 2 weeks) hiPSCRPE cultures showed increased MMP-1 activity in CSE-treated hiPSC-RPE cultures. Data are presented as mean \pm SEM, $P=0.06, n=3$ independent trials. $\mathbf{b}$ Spectrometric measurement of cell culture media at distinct time points $(0 \mathrm{~h}, 4 \mathrm{~h}, 8 \mathrm{~h}, 12 \mathrm{~h}$, and $24 \mathrm{~h})$ during a $24 \mathrm{~h}$ period after addition of CSE $(0 \mathrm{~h})$ to the media showed constant cigarette smoke level as measured by absorbance at $320 \mathrm{~nm}^{71}$. c-e Representative western blot images (c) and corresponding quantitative analyses (d, e) showing similar uptake $(0 \mathrm{~h})(\mathbf{c}, \mathbf{d})$ and degradation $(24 \mathrm{~h})(\mathbf{c}, \mathbf{e})$ of RHO/POS in untreated versus CSEtreated (0.5\%) hiPSC-RPE cultures. Data are presented as mean \pm SEM, $n=5$ independent trials. Note: ACTN served as loading control for western blot analyses. $\mathbf{f}$ Representative fluorescent microscopy (top panel) and confocal microscopy images (bottom panel) displaying similar cell viability (calceinAM) and ZO-1 localization in untreated versus CSE-treated $(0.5 \%, 24 \mathrm{~h}$ ) hiPSC-RPE cultures (scale bars $=20 \mu \mathrm{m})$. $\mathbf{g}$ TER measurements of untreated versus CSE-treated $\left(0.5 \%, 24 \mathrm{~h}\right.$ ) hiPSC-RPE were similar at baseline and the $24 \mathrm{~h}$ time point with TER $\geq 150 \Omega \mathrm{cm}^{2}$ (dotted line, the reported in vivo TER threshold ${ }^{40}$ ). Data are presented as mean \pm SEM. $n=3$ independent trials

RPE viability or tight junction integrity as evaluated by live-cell staining (Fig. 5a), ZO-1 staining (Fig. 5b) and TER measurements (Fig. 5c). We next investigated whether continuous exposure to excess Fe and/or cigarette smoke over a chronic (2 weeks) timeframe exacerbated autofluorescent material (POS digestion products) accumulation in hiPSC-RPE cells. Specifically, autofluorescence accumulation (ex: $546 \mathrm{~nm}$, em: $560-615 \mathrm{~nm}$ ) consistent with lipofuscin accumulation ${ }^{50,51}$ was compared to POSfed untreated versus POS-fed FAC + CSE-treated hiPSCRPE cultures. Confocal microscopy analyses of autofluorescence levels revealed increased accumulation of autofluorescent material in POS-fed FAC + CSE-treated hiPSC-RPE compared to only POS-fed untreated hiPSCRPE cultures (Figs. $5 \mathrm{~d}$ and $6 \mathrm{a}-\mathrm{c}$ and Supplementary Table 2). Specifically, quantitative analyses of autofluorescence levels in three distinct trials revealed both (i) a greater number of autofluorescent particles per 100 RPE cell nuclei (Fig. 6a, b and Supplementary Table 2) and (ii) an increased area of autofluorescence per $100 \mathrm{RPE}$ cell nuclei in POS-fed FAC + CSE-treated hiPSC-RPE cultures when compared to untreated hiPSC-RPE cultures (Fig. 6a, c and Supplementary Table 2). Of note, utilizing orthogonal view of autofluorescence in RPE whole mounts immunostained for cell nuclei (Hoechst) and tight junction protein (ZO-1), we also confirmed the intracellular pattern of autofluorescent material accumulation in both POS-fed and untreated and POS-fed and FAC + CSE-treated hiPSC-RPE cultures (Fig. 5d, bottom panel). Altogether, these results demonstrate that in the presence of a physiologically relevant stressor, daily POS feeding, exposure of hiPSC-RPE cultures to AMD-relevant stressors, excess $\mathrm{Fe}$, and cigarette smoke, is sufficient to cause increased autofluorescent material accumulation, a known pathological hallmark linked to AMD development ${ }^{50}$.

\section{Discussion}

RPE cell dysfunction in the retina plays a central role in several retinal degenerative diseases, including $A M D^{52-54}$. Although inhaled smoke and altered Fe metabolism have been implicated in AMD development and progres$\operatorname{sion}^{2,4,7,8,47}$, the precise impact of cigarette smoke and/or excess Fe on human RPE cells and its consequence for the development of AMD-relevant pathological changes have not been established. In this study, we utilized FAC and CSE to mimic Fe and cigarette smoke exposure in hiPSCRPE cells and showed that, in the absence of any genetic defect, these AMD-associated risk factors can alter a key RPE function, phagocytosis and degradation of shed POS, and subsequently lead to increased accumulation of autofluorescent material within the RPE cells. Specifically, our data show a stressor-specific impact of FAC and/or CSE on POS processing in hiPSC-RPE cells with (i) reduction in POS uptake after acute exposure to FAC or FAC + CSE (Figs. 2d, e and 4a, b) and (ii) decrease in POS degradation following combined exposure to FAC + CSE (Fig. 4a, c). Notably, reduced POS degradation in FAC + CSE-treated hiPSC-RPE cultures correlates with decreased levels of active CTSD (Fig. 4d, e), a lysosomal enzyme involved in POS digestion. Furthermore, consistent with impaired POS degradation, chronic exposure (2 weeks) to FAC + CSE in the presence of daily POS feeding is sufficient to elicit increased accumulation of autofluorescent material (plausibly POS digestion products), in hiPSC-RPE cells (Figs. 5, 6).

In numerous retinal degenerative diseases that affect the outer retina, impairment of a specific RPE function, phagocytosis and degradation of shed POS contributes to the disease development. For instance, impaired POS uptake by RPE cells is causal in RP, harboring mutations in the MERTK gene ${ }^{38,39}$. Similarly, impaired POS processing by RPE cells has been linked to Stargardt disease pathology ${ }^{55-57}$, inherited maculopathies like $\mathrm{BD}^{36,37,58}$ and $\mathrm{AMD}^{59,60}$. It is noteworthy that a commonality in the pathology of these distinct diseases is the accumulation of autofluorescent material, lipofuscin (POS-breakdown products), in the retina/RPE layer of affected patient eyes. Apart from genetic defects, aging, the single biggest risk factor associated with AMD development, supports increased accumulation of autofluorescent POS- 
a

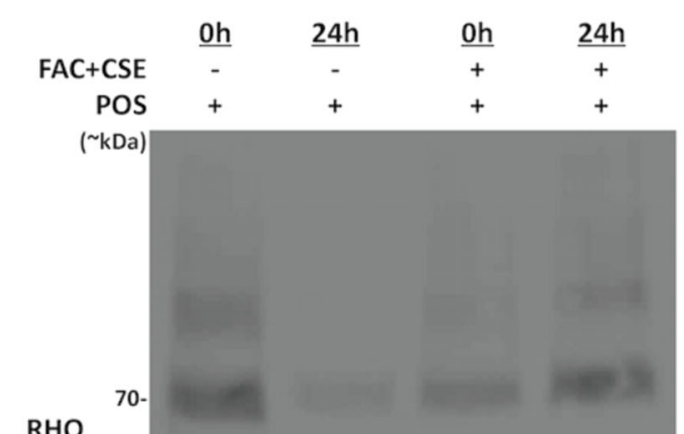

ACTN

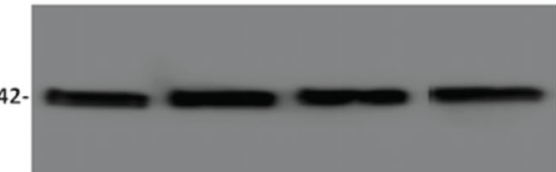

d

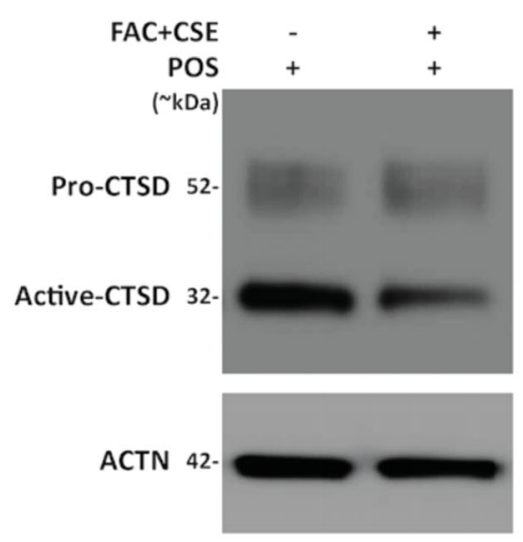

f
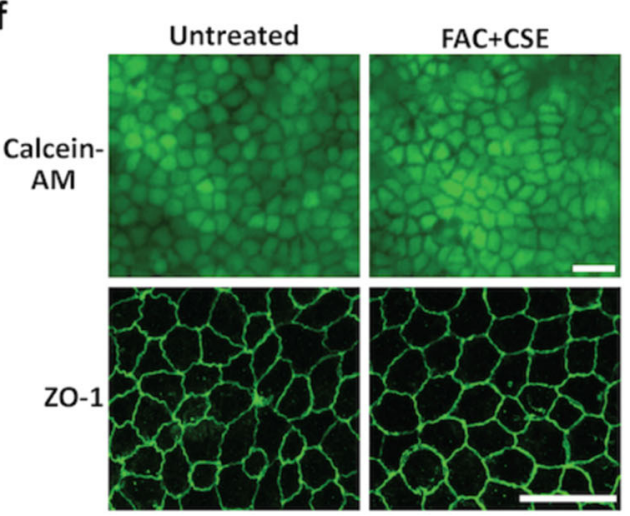

b



C

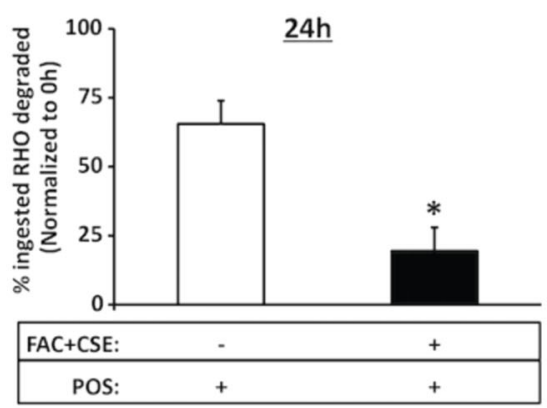

e

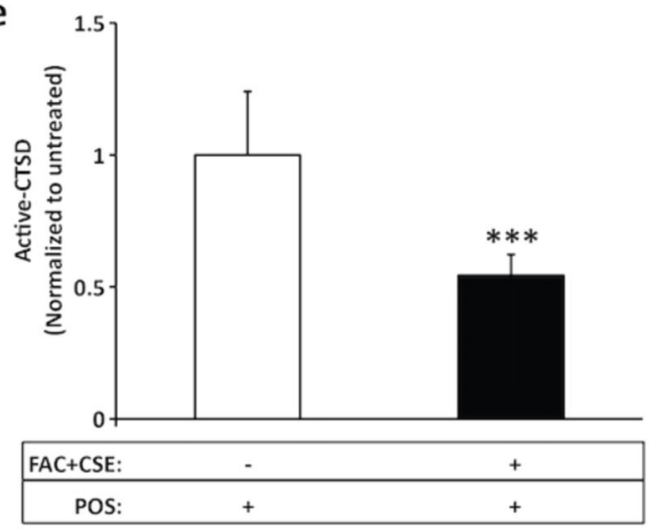

g

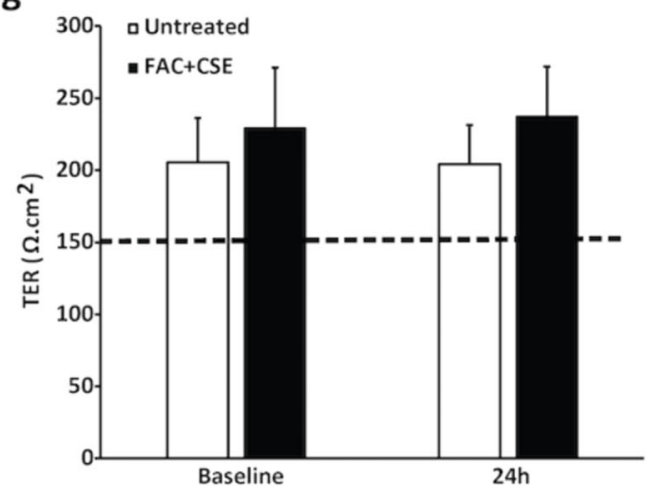

Fig. 4 (See legend on next page.) 
(see figure on previous page)

Fig. 4 Combined supplementation of FAC and CSE to the culture media is sufficient to decrease both uptake and degradation of POS by hiPSC-RPE cells. a-c Representative western blot images (a) and corresponding quantitative analyses $(\mathbf{b}, \mathbf{c})$ showing reduced uptake of RHO/POS at the $0 \mathrm{~h}$ time point $(\mathbf{a}, \mathbf{b})$ and decreased degradation of ingested RHO/POS at $24 \mathrm{~h}$ time point $(\mathbf{a}, \mathbf{c})$ in untreated versus FAC + CSE-treated $(200 \mu \mathrm{gg} / \mathrm{ml}$ $+0.5 \%)$ hiPSC-RPE cultures. Data are presented as mean $\pm \mathrm{SEM}, n=3$ independent trials. ${ }^{*} P \leq 0.05$. ACTN served as loading control. $\mathbf{d}-\mathbf{e}$

Representative western blot images (d) and corresponding quantitative analyses (e) displaying reduced levels of active CTSD (32 kDa) in POS-fed and FAC + CSE-treated hiPSC-RPE cultures compared to only POS-fed hiPSC-RPE cultures. Data are presented as mean \pm SEM, $n=3$ independent trials. ${ }^{*} P \leq 0.05,{ }^{* * *} P \leq 0.001$. Note: ACTN served as loading control for western blot analyses. $\mathbf{f}$ Representative fluorescent microscopy (top panel) and confocal microscopy images (bottom panel) demonstrating equivalent staining of calcein-AM-positive live cells (top panel) and ZO-1 localization (bottom panel) in untreated and FAC + CSE-treated $(200 \mu \mathrm{g} / \mathrm{ml}+0.5 \%, 24 \mathrm{~h})$ hiPSC-RPE cultures (scale bar $=20 \mu \mathrm{m}) . \mathbf{g}$ TER $\geq 150 \Omega \mathrm{cm}^{2}$ was seen at both baseline and $24 \mathrm{~h}$ time point in untreated and FAC + CSE-treated $(200 \mu \mathrm{g} / \mathrm{ml}+0.5 \%, 24 \mathrm{~h})$ hiPSC-RPE cultures. Data are presented as mean \pm SEM. $n=3$ independent trials

a

POS fed (2wk)

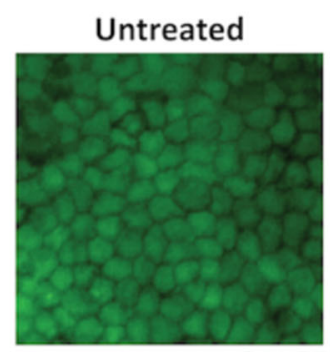

CALCEIN-AM

C

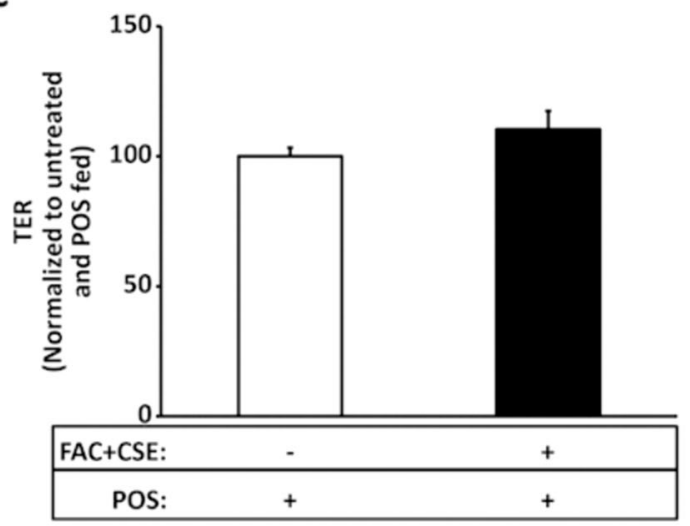

b

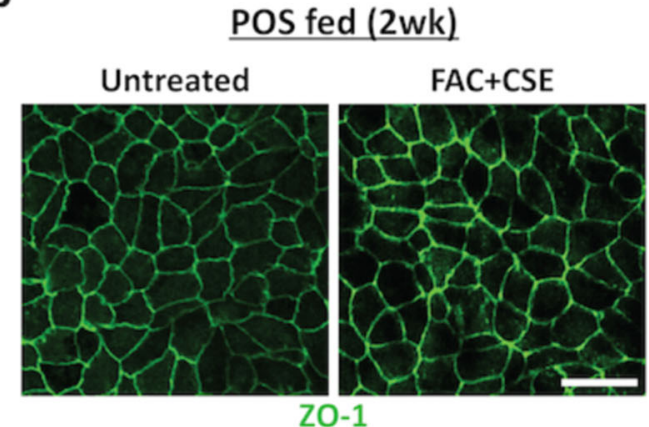

d

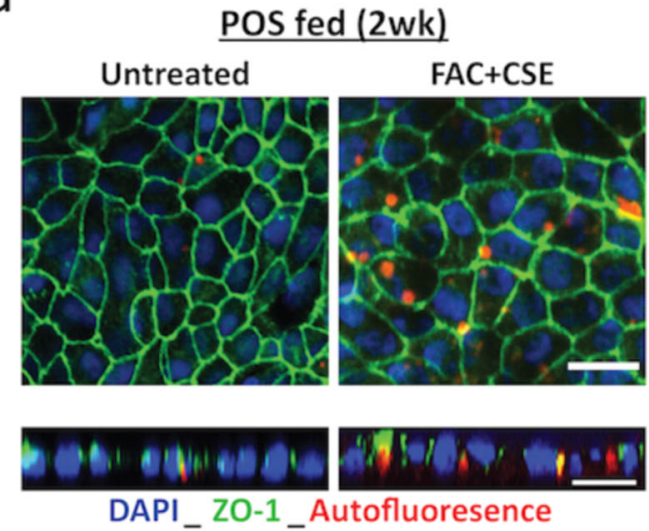

Fig. 5 FAC + CSE supplementation of culture media over 2 weeks leads to intracellular accumulation of autofluorescent material in daily POS-fed hiPSC-RPE cultures. a Representative fluorescence microscopy images showing similar cell viability (calcein-AM) in FAC + CSE-treated $(200 \mathrm{\mu g} / \mathrm{ml}+0.5 \%)$ and POS-fed (20 POS/RPE/day) and untreated but POS-fed (20 POS/RPE/day) hiPSC-RPE cultures after 2 weeks of daily POS feeding and FAC + CSE supplementation to the culture media. Of note, untreated cultures had media change with regular cell culture media during daily POS feeding (scale bar $=25 \mu \mathrm{m}$ ). b Representative confocal images depicting similar tight junction morphology (ZO-1) in untreated but POS-fed (daily for 2 weeks) and FAC + CSE-treated $(200 \mu \mathrm{g} / \mathrm{ml}+0.5 \%$, daily for 2 weeks) and POS-fed (20 POS/RPE/day for 2 weeks) hiPSC-RPE cultures. (Scale bar $=25 \mu \mathrm{m})$. c TER measurements showing similar TER values in POS-fed and FAC + CSE-treated $(200 \mu \mathrm{g} / \mathrm{ml}+0.5 \%)$ and untreated but POS-fed hiPSC-RPE cultures after 2 weeks of daily POS feeding and FAC + CSE supplementation. Data are presented as mean \pm SEM, $n=3$ independent trials. d Representative confocal microscopy images at the 2 weeks time point showing increased accumulation of autofluorescent material in spectral wavelength (ex: $546 \mathrm{~nm}$, em: 560-615 nm) consistent with lipofuscin accumulation (top panel) in FAC + CSE-treated and POS-fed hiPSC-RPE cultures compared to untreated but POS-fed hiPSC-RPE cultures. Furthermore, orthogonal view of hiPSC-RPE cultures comparing the localization of autofluorescence accumulation (red) in relationship to tight junction marker ZO-1 (green) and Hoechst-stained cell nuclei (blue) confirming the intracellular localization of autofluorescent particles in both untreated and FAC + CSE-treated POS-fed cultures (bottom panel). (Scale bar $=25 \mu \mathrm{m}$ ) 


\section{POS fed (2wk)}

a

Trial 1
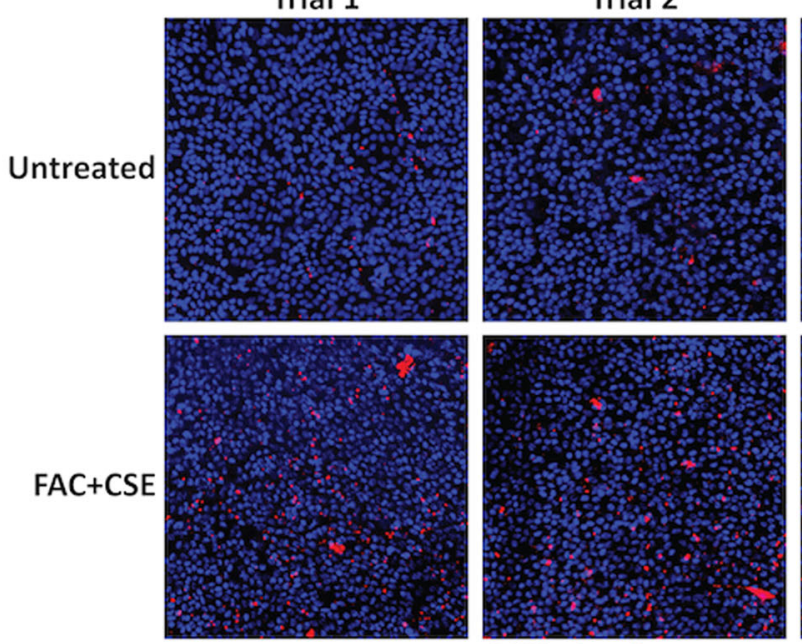

DAPI_Autoflourescence

Trial 3
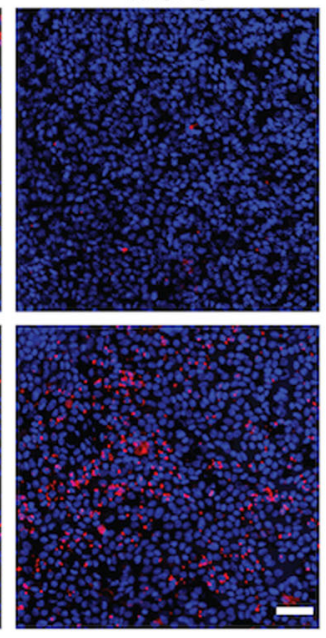

$$
\text { (1) }
$$

b

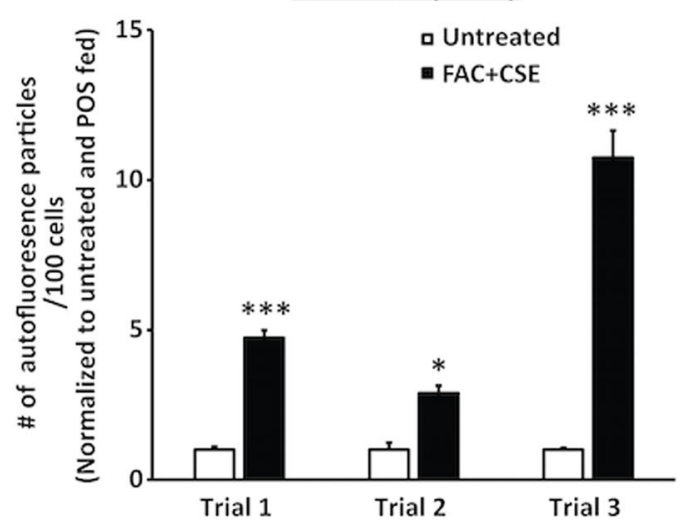

C

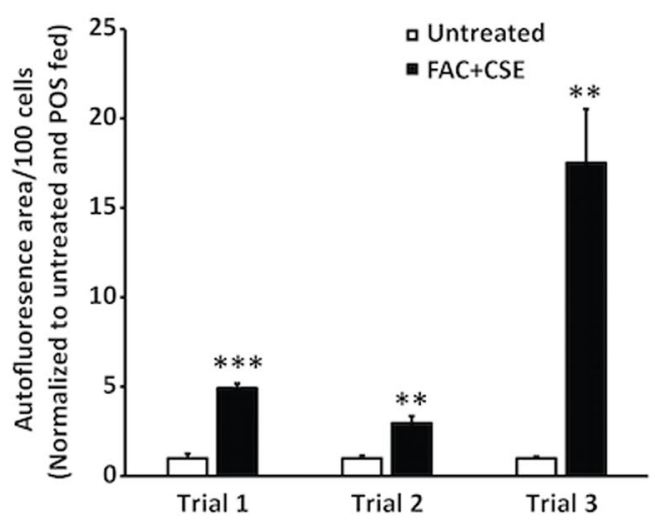

Fig. 6 Chronic supplementation of FAC and CSE to culture media over 2 weeks leads to both increased number and area of autofluorescent particles in daily POS-fed hiPSC-RPE cultures. a Representative confocal images from three distinct trials showing increased accumulation of autofluorescent particles (red; spectral wavelength ex: $546 \mathrm{~nm}$, em: $560-615 \mathrm{~nm}$ ) in FAC + CSE-treated (200 $\mu \mathrm{g} / \mathrm{ml}+0.5 \%)$ and daily POS-fed (20 POS/RPE/cell) hiPSC-RPE cultures compared to untreated but daily POS-fed hiPSC-RPE cultures. Of note Hoescht-stained RPE cell nuclei are seen in blue. b-c Quantitative analysis of autofluorescence in untreated and POS-fed hiPSC-RPE cultures versus POS-fed FAC + CSE-treated hiPSCRPE cultures in three distinct trials showed both increased number (c) and area (d) of autofluorescent particles in POS-fed FAC + CSE-treated hiPSCRPE cultures in each individual trial. Data are presented as mean \pm SEM. ${ }^{* *} P \leq 0.001,{ }^{* *} P \leq 0.01,{ }^{*} P \leq 0.05 . n=3$ independent trials

breakdown products within the RPE cells ${ }^{50}$. In fact, agingassociated increases in RPE autofluorescence have been linked to RPE cell death ${ }^{24}$ and development of AMD pathology ${ }^{50}$. Interestingly, the RPE cell monolayer is likely to be exposed to both Fe overload and cigarette smoke with aging. In support, aging-associated increases in RPE Fe levels have been reported in both rodent models ${ }^{61}$ and human subjects $^{8,9}$. Furthermore, cigarette smoke exposure is a lifestyle risk factor that is more prevalent in adults, increasing with age in the age group of $18-64$ years $^{19}$. Notably, Fe and cigarette smoke have been shown to affect cellular compartments/pathways involved in POS processing by RPE cells ${ }^{6,29,30,32}$. Taken in the context of our results showing combined $\mathrm{Fe}$ and cigarette smoke exposure in control hiPSC-RPE is sufficient to reduce active-CTSD levels, impair POS digestion and cause increased autofluorescent material accumulation, these data raise questions about the contribution of cell intrinsic (e.g., Fe overload) and extrinsic (e.g., cigarette smoke) stressors in the increased autofluorescence seen with aging in the RPE monolayer in vivo ${ }^{53}$. 
It is plausible that changes in POS processing and autofluorescence accumulation observed in our study after FAC + CSE exposure were aided by the fact that hiPSC-RPE cells can be cultured over an extended period of time and this allowed us to use "aged" ( 60-90 days in culture) hiPSC-RPE cells in our experiments. This is especially relevant given the fact that in other disease modeling studies, "aging" of hiPSC-RPE cells plausibly contributed to the development of specific human disease phenotype(s), like drusen formation ${ }^{34}$ and autofluorescent material accumulation in culture ${ }^{36}$. Another advantage of hiPSC-based disease modeling utilized in the current study was the ability to select (i) hiPSCs/human subjects and (ii) hiPSC-derived target cell fitting the specified criteria. To evaluate the singular role of $\mathrm{Fe}$ and/or cigarette smoke in RPE cell function specifically, we chose control hiPSC-RPE lines that did not harbor a known gene-defect making them susceptible to MD. Furthermore, the ability to use multiple $(n \geq 3)$ hiPSC control lines allowed us to introduce biologic variability in a cell culture model assessing the impact of extrinsic stressors on RPE phagocytosis and degradation. On the flip side, we did not pursue the previously shown consequences of autofluorescence accumulation (e.g., RPE cell death ${ }^{62}$, increased oxidative stress ${ }^{63}$ ) in hiPSC-RPE cultures. This would be an important consideration for future studies, especially given the fact that presence of Fe overload and/ or cigarette smoke exposure could interact with and further exacerbate the consequences of increased autofluorescent POS-digestion by-products in RPE cells. For instance, a recent study has shown a role of Fe in POSmediated RPE dysfunction through Fe interactions with bisretinoids in lipofuscin supporting RPE cell damage ${ }^{64}$. The long-term impact of Fe and cigarette smoke on POS uptake and digestion is also of future interest in that we limited our FAC/CSE/FAC + CSE exposure to acute $(24 \mathrm{~h})$ experiments when evaluating the rate of POS degradation. It is plausible that extended exposure to FAC or CSE by itself would have been sufficient to reduce active-CTSD levels and decrease POS degradation and thereby increase autofluorescent material accumulation in hiPSC-RPE cells. However, utilizing FAC, CSE, and FAC + CSE supplementation over acute $(24 \mathrm{~h})$ timeframe, helped highlight potentially more detrimental consequences of multiple simultaneous environmental stressors, a logical but yet untested scenario with regard to RPE cell dysfunction and AMD development.

In summary, we report that excess Fe and cigarette smoke alone and in combination can impair POS processing plausibly by affecting lysosomal function and lead to increased autofluorescent material accumulation in control hiPSCRPE. This has important implications for the causative role of cell intrinsic and extrinsic stressors in instigating RPE cell dysfunction in retinal degenerative diseases, like AMD.

\section{Materials and methods}

\section{Generation and characterization of hiPSCs}

Collection of patient samples and subsequent experimental analyses were performed in accordance with University of Rochester Institutional Regulatory Board approval (RSRB00056538) and conformed to the requirements of the National Institutes of Health and Declaration of Helsinki. Skin fibroblasts from individuals with no known history of retinal disease were reprogrammed into hiPSCs lines using a previously described protocol $^{34,65}$. Briefly, fibroblasts were maintained in fibroblast medium containing DMEM with high glucose, $10 \%$ fetal bovine serum, GlutaMAX ${ }^{\mathrm{TM}}$ and $100 \mathrm{U} / \mathrm{mL}$ penicillin-streptomycin (Thermo Fisher Scientific, Waltham, MA, USA). iPSCs were generated by reprogramming the fibroblasts with nonintegrating episomal vectors containing six reprogramming factors (OCT-4, SOX2, KLF4, L-MYC, LIN28, and shRNA for p53) as described previously ${ }^{65}$. Reprogramming was initiated by using the nucleofection kit for primary fibroblasts (Lonza) and nucleofected using the Nucleofector 2b Device (Lonza, Program T-016) by transfecting 60,000 fibroblasts with $1 \mu \mathrm{g}$ each of the following plasmids: pCXLE-hOCT4shP53, pCXLE-hSK and pCXLE-hUL (Addgene plasmids \#27077, 27078, 27080). Transfected cells were then maintained and grown in fibroblast medium for 6 more days following trypsinization and plating $1 \times 10^{5}$ cells onto a $10 \mathrm{~cm}$ dish that was previously seeded with irradiated mouse embryonic fibroblast (MEF) feeder layer (MTIGlobalStem, Thermo Fisher Scientific). The following day, the medium was changed to hiPSC medium (DMEM/F12 with $20 \%$ Knockout serum replacement, 1\% MEM-NEAA, $1 \%$ GlutaMAX $^{\mathrm{TM}}$ and $100 \mathrm{ng} / \mathrm{mL}$ FGF2). At days 17-30 from transfection, iPSC colonies started appearing and were manually picked and expanded for further characterization. Multiple hiPSC clones were generated, isolated and characterized for expression of pluripotency markers (OCT3/4, NANOG) for every control fibroblast sample by immunocytochemical analysis as previously described $^{34,36,66,67}$. The reprogrammed iPSC lines were maintained undifferentiated on Matrige ${ }^{\infty}$ (Corning, Corning, NY, USA) and supplemented with mTESR $^{\mathrm{TM}} 1$ (STEMCELL Technologies, Vancouver, Canada) or maintained on MEFs and supplemented with hiPSC medium. For all hiPSC lines utilized in this study, pluripotency characterization has been previously published $^{34,67}$.

\section{Differentiation and culture of hiPSC-RPE}

hiPSCs were grown either on MEF feeder layer or on Matrigel $^{\circledast}$ as described previously ${ }^{68,69}$. Differentiation of hiPSCs to obtain RPE was carried out using previously established protocols ${ }^{69,70}$. RPE cells in these adherent differentiated hiPSC cultures ${ }^{68}$ were allowed to mature for 
a total of 60-90 days prior to passaging and plating onto nonpermeable plastic support (24-well plates) to yield passage 1 (P1) hiPSC-RPE monolayers ${ }^{36,68}$. Mature monolayers of P1 hiPSC-RPE on 24-well plates were terminally passaged onto either Transwell "inserts (CoStar, Corning) or 24-well or 6-well plates using the above described protocol to obtain mature monolayers of P2 hiPSC-RPE cells ${ }^{36,68}$. Unless stated otherwise, parallel age-matched hiPSC-RPE monolayers on transwells and/or 24- and 6-well plates at P2 were used in all experiments.

\section{Preparation and characterization of cigarette smoke extract}

CSE was prepared by bubbling smoke into $20 \mathrm{ml}$ of serum-free RDM using 1R3F research grade cigarettes (Kentucky Tobacco Research Council, Lexington, KT, USA $)^{71,72}$. The optical density of freshly prepared CSE was measured at a wavelength of $320 \mathrm{~nm}$ to characterize and normalize its strength. Of note, an optical density of 0.65 is considered to represent $100 \% \mathrm{CSE}^{71}$. Furthermore, CSE was prepared fresh daily for single use, sterile filtered using $0.22 \mu \mathrm{m}$ Millex syringe filters (EMD Millipore, Billerica, MA, USA) and diluted to the desired concentration in serum-free RPE cell culture maintenance media (RDM).

\section{Acute and chronic supplementation of hiPSC-RPE cultures with FAC and CSE}

The amount of FAC and CSE utilized in this study to assess the acute $(24 \mathrm{~h}, \mathrm{FAC}$ at $200 \mu \mathrm{g} / \mathrm{ml}, \mathrm{CSE}$ at $0.5 \%)$ and chronic ( 2 weeks to 1 month, FAC at 50 and $200 \mu \mathrm{g} / \mathrm{ml}$, CSE at 0.5\%) impact of excess Fe and CSE was based on previously published studies in primary RPE cell cultures (mice and human fetal RPE), and immortalized cell lines (HepG2 and ARPE-19) $)^{5,41-44}$. Specifically, hiPSC-RPE cultures were supplemented with FAC (50 and $200 \mu \mathrm{g} /$ $\mathrm{ml})$, CSE $(0.5 \%)$, or FAC + CSE $(200 \mu \mathrm{g} / \mathrm{ml}+0.5 \%)$ in the cell culture maintenance media (RDM) in acute $(24 \mathrm{~h})$ and chronic ( 2 weeks to 1 month) experiments. Of note, hiPSC-RPE cells from parallel cultures serving as untreated controls were fed the same amount of RDM alone on a daily basis. In chronic experiments, lasting 2 weeks to 1 month, media of untreated hiPSC-RPE cultures and FAC and CSE and/or FAC + CSE-treated hiPSC-RPE cultures were replaced on a daily basis. Furthermore, in experiments evaluating phagocytosis and degradation of POS and autofluorescence accumulation post-POS feeding, untreated hiPSC-RPE cultures were fed $\mathrm{RDM}$ and POS (20 POS/RPE/day) whereas treated hiPSCRPE cultures were fed RDM supplemented with FAC, CSE, and FAC + CSE and the same amount of POS during daily media change. Of note, media change and daily POS feeding in these experiments were performed at the same time each day.

\section{Measurement of TER}

TER of hiPSC-RPE monolayers on transwell inserts was measured using an epithelial volt-ohm meter (EVOM2, World Precision Instruments, Sarasota, FL, USA) following manufacturer's instructions as previously described ${ }^{73}$. In $24 \mathrm{~h}$ acute experiments, TER was measured once at baseline before the hiPSC-RPE cells were subjected to FAC, CSE, and/or FAC + CSE treatments, and at the end of the experiment at the $24 \mathrm{~h}$ time point. For chronic experiments, lasting 2 weeks, baseline TER was measured at day 0 prior to the start of treatments and subsequently at day 14. Of note, in accordance with other published studies, TER measurements reported in this study were calculated by multiplying blank subtracted raw TER values with the surface area of the transwell filters ${ }^{68}$.

\section{Assessment of cell viability}

Calcein-AM (Life Technologies, Carlsbad, CA, USA) was used to determine the cell viability in accordance with the manufacturer's protocol. Specifically, hiPSC-RPE cultures were incubated in the dark with calcein-AM $(5 \mu \mathrm{M})$ and nuclear staining dye (Hoechst, 1:2000; Life Technologies) for $30 \mathrm{~min}$ at $37^{\circ} \mathrm{C}$. Calcein-AM and Hoechst-stained hiPSC-RPE samples were imaged on a Leica DM IRBE inverted microscope (Leica Microsystems, Buffalo Grove, IL, USA) using a Lumenera INFINITY3-1 camera and INFINITY ANALYZE software (Lumenera, Ontario, Canada).

\section{Prussian blue staining}

hiPSC-RPE cultures were incubated with equal parts of $20 \%$ aqueous solution of hydrochloric acid $(\mathrm{HCl})$ and $10 \%$ aqueous solution of $\mathrm{K}_{4}\left[\mathrm{Fe}(\mathrm{CN})_{6}\right]_{4-}$ for $20 \mathrm{~min}$. hiPSCRPE cells were then washed three times with double distilled water $\left(\mathrm{ddH}_{2} \mathrm{O}\right)$ for 5 min each and immediately imaged using a Motic moticam2000 color camera (Kowloon, Hong Kong).

\section{Quantitative real-time PCR}

As described previously ${ }^{34}$, total RNA was extracted using the RNeasy Mini Plus Kit and DNase I treatment was performed to digest any residual genomic DNA (Qiagen, Germantown, MD). cDNA was synthesized using the iScript cDNA Synthesis Kit (Bio-Rad, Hercules, CA) following the manufacturer's instructions. Quantitative real-time PCR (qRT-PCR) experiments were carried out using gene-specific primers (Supplementary Table. 3) and the Sso Advanced SYBR Green Supermix (Bio-Rad) in a Bio-Rad CFX Thermal cycler (40 cycles). Data were 
analyzed using Bio-Rad CFX software (Bio-Rad) and Microsoft Excel.

\section{Phagocytosis and degradation of POS}

Age-matched mature monolayers of hiPSC-RPE cells on transwell inserts were utilized to evaluate phagocytosis and degradation of POS at $0 \mathrm{~h}$ and $24 \mathrm{~h}$ respectively using a previously described protocol ${ }^{36,58}$. Briefly, hiPSC-RPE cultures were fed unlabeled POS (20 POS/RPE cell; Invision Bioresources, Seattle, WA, USA) for $2 \mathrm{~h}$. At the end of the $2 \mathrm{~h}$ incubation, POS containing media was removed and RPE cells were washed vigorously five times with $1 \mathrm{X}$ phosphate buffer saline (PBS) to remove any unbound POS remaining on the RPE cell surface. RPE cells were either immediately harvested ( $0 \mathrm{~h}$ time point) or continued in culture and harvested at the $24 \mathrm{~h}$ time point for analyses by western blotting. Specifically, the levels of a POS-specific protein, RHO (1:500, EMD Millipore) relative to loading control ACTN (1:500, Santa Cruz Biotechnology, Dallas, TX, USA) were determined by quantitative western blotting to assess the uptake of POS $(0 \mathrm{~h})$ and rate of degradation of ingested POS $(24 \mathrm{~h})$. Of note, monomer $(\sim 35 \mathrm{kDa})$, dimer $(\sim 70 \mathrm{kDa})$ and multimer bands $(>70-250 \mathrm{kDa})$ and plausibly glycosylated forms $(40 \mathrm{kDa})$ of RHO were all considered for RHO quantification for western blot analyses. Furthermore, when testing the effect of specific stressors (FAC and/or CSE) on phagocytosis/degradation, hiPSC-RPE cultures were treated with FAC $(200 \mu \mathrm{g} / \mathrm{ml})$ and/or CSE $(0.5 \%)$ concurrently with POS feeding (20 POS/RPE cell). In addition, when cell culture media were replaced after $2 \mathrm{~h}$ POS feeding, the fresh cell culture media were supplemented with the appropriate stressor (FAC, CSE, or FAC + CSE).

\section{Zymography analyses for MMP-1 activity}

MMP-1 activity was analyzed by performing zymography on conditioned media obtained from the basal chamber of hiPSC-RPE cultures on transwells in accordance with a previously described protocol ${ }^{74}$. Briefly, conditioned media samples were electrophoresed on $10 \%$ polyacrylamide gels containing $1 \mathrm{mg} / \mathrm{ml}$ collagen (SigmaAldrich). Subsequently, the polyacrylamide gels were incubated in $2.5 \%$ Triton-X-100 in $1 \mathrm{X}$ PBS for a period of $2 \mathrm{~h}$. After three additional rinses, the gels were incubated in prewarmed MMP-1 incubation buffer $(50 \mathrm{mM}$ Tris$\mathrm{HCl}, \mathrm{pH}, 7.4,5 \mathrm{mM} \mathrm{CaCl}_{2}, 0.001 \% \mathrm{NaN}_{3}, 0.005 \%$ Triton$\mathrm{X}-100, \mathrm{pH} 7.75)$ for $36 \mathrm{~h}$ at $37^{\circ} \mathrm{C}$. Following a brief rinse in destaining solution (1:3:6 acetic acid:methanol:water), the gel was stained with $0.2 \%$ Coomassie blue solution for $2-3 \mathrm{~h}$ at room temperature. Finally, the gels were rinsed again in destaining solution and imaged on Azure C500 imaging system (Azure Biosystems, Dublin, CA, USA). Of note, quantitative analyses were carried out using Image Studio Lite Version 5.2 and Microsoft Excel.

\section{Quantitative western blot analyses}

hiPSC-RPE cells were lysed and an equivalent microgram load of protein from untreated and treated samples were resolved on 4-20\% Tris- $\mathrm{HCl}$ gradient gels (Bio- $\mathrm{Rad}$ ) and transferred onto low fluorescence polyvinylidene difluoride (PVDF) membranes (Bio-Rad) as previously described $^{34}$ and probed with the following primary antibodies: RHO, ACTN, or CTSD (1:500, Santa Cruz Biotechnology). Secondary antibodies utilized were nearInfrared fluorescent (1:10,000, Licor Biosciences) or peroxidase-conjugated secondary antibodies (1:10,000, Azure Biosystems). The blots were either imaged on Odyssey Infrared Imager (Licor Biosciences) or developed using the Radiance Plus Chemiluminescence Kit and imaged on Azure C500 imaging system (Azure Biosystems). Of note, prior to reprobing with a different antibody, the PVDF membrane was stripped using NewBlot PVDF stripping buffer (Licor Biosciences) for $60 \mathrm{~min}$ at room temperature. Quantitative analysis of all the western blotting data was carried out using the image acquisition software (Licor Odyssey 3.0 and/or Image Studio Lite version 5.2) and Microsoft Excel.

\section{Immunocytochemical analyses}

Immunocytochemistry of hiPSC/hiPSC-RPE cultures was performed using a previously published protocol ${ }^{34,67}$. Briefly, cells were fixed in $4 \%$ paraformaldehyde for $30 \mathrm{~min}$ at $4{ }^{\circ} \mathrm{C}$ followed by blocking in $1 \mathrm{X}$ blocking buffer $[10 \%$ normal donkey serum (ImmunoReagents Inc., Raleigh, NC, USA) and $0.1 \%$ Triton-X-100 in $1 \mathrm{X} \mathrm{PBS]} \mathrm{for}$ $1 \mathrm{~h}$. This was followed by incubation in a primary antibody solution with $0.5 \mathrm{X}$ blocking buffer at $4{ }^{\circ} \mathrm{C}$ overnight. The samples were washed two times in $0.05 \%$ Triton-X100 in 1X PBS the following day and incubated in hostspecific secondary antibodies diluted with $0.5 \mathrm{X}$ blocking buffer for $1 \mathrm{~h}$ at room temperature. Samples were then washed twice in $0.05 \%$ Triton-X-100 in $1 \mathrm{X}$ PBS, incubated with nuclear staining dyes (DAPI or Hoechst; Life Technologies) for $15 \mathrm{~min}$ in PBS and coverslipped in Prolong gold (Life Technologies). Of note, primary antibodies used for immunocytochemical analysis included EZR (1:100, Cell Signaling Technology, Danvers, MA) and ZO-1 (1:100, Life Technologies). Secondary antibodies used in this study were Alexa Fluor-conjugated (Life Technologies) at a concentration of 1:500. Image acquisition was carried on a confocal microscope (LSM 510 META, ZEISS, Thornwood, NY, USA) using Zen 2009 software (ZEISS).

\section{Quantification of autofluorescence in POS-treated cultures}

hiPSC-RPE cultures post chronic (2 weeks) POS feeding were washed five times with $1 \mathrm{X}$ PBS to remove any unbound POS remaining on the RPE cell surface. Subsequently immunocytochemical analyses (as described 
above) were performed to localize ZO-1 and DAPI. After image acquisition, autofluorescence in excitation $(543 \mathrm{~nm})$ and emission $(560-615 \mathrm{~nm})$ spectra consistent with lipofuscin were evaluated in $n=6$ random images per sample per trial using Image software $(\mathrm{NIH})$ and plotted as (i) the number of autofluorescence particles and (ii) total autofluorescent area per 100 cells (as measured by DAPI-stained nuclei).

\section{Statistics}

All experiments were performed on at least $n \geq 3$ hiPSCRPE cultures obtained from independent hiPSCdifferentiation runs and data throughout the manuscript are expressed as mean \pm SEM. Furthermore, statistical significance $(P \leq 0.05)$ was evaluated using an unpaired two-tailed Student's t-test in Microsoft Excel.

\begin{abstract}
Acknowledgments
The authors would like to acknowledge the access to Inhalation Core Facility at the University of Rochester to prepare CSE, and Susan Messing and Bokai Wang from the Department of Biostatistics and Computational Biology at University of Rochester for their consulting services in statistical analyses. Electron microscopy was completed at the University of Rochester Medical Center Electron Microscope Shared Resource Laboratory. This work was supported by the BrightFocus Foundation Macular Degeneration Grant (to R. S.), David Bryant Trust (to R.S.), Foundation of Fighting Blindness Individual Investigator Award (to R.S.), National Institute of Health, NIH-1R01EY028167 (to R.S.), Retina Research Foundation and Research to Prevent Blindness, RPB's Career Development Award (to R.S.), Unrestricted Challenge Grant to Department of Ophthalmology at University of Rochester.
\end{abstract}

\section{Author details}

'Department of Ophthalmology (Flaum Eye Institute), University of Rochester, Rochester, NY, USA. ²Department of Biomedical Genetics, University of Rochester, Rochester, NY, USA. ${ }^{3}$ UR Stem Cell and Regenerative Medicine Institute, Rochester, NY, USA. ${ }^{4}$ Center for Visual Science, University of Rochester, Rochester, NY, USA. ${ }^{5}$ Present address: Department of Pathology and Lab Medicine, University of Rochester, Rochester, NY, USA

\section{Authors' contributions:}

Conceptualization, S.D., C.A.G., and R.S.; data curation, S.D. and C.A.G.; formal analysis, S.D., C.A.G., and C.T.; funding acquisition, R.S.; investigation, S.D., C.A.G., L.W., A.H., C.S., L.A.M., and R.S.; supervision, R.S.; writing--original draft, S.D. and R.S.; writing--review and editing, S.D., C.A.G., and R.S. All the authors have read and approved the final manuscript.

\section{Conflict of interest}

The authors declare that they have no conflict of interest.

\section{Abbreviations}

RPE, Retinal pigment epithelium; POS, Photoreceptor outer segments; AMD, Age-related macular degeneration; hiPSC, Human induced pluripotent stem cell; CSE, Cigarette smoke extract; RP, Retinitis pigmentosa; B(a)P, Benzo(a) Pyrene; TER, Transepithelial resistance; SFD, Sorsby's fundus dystrophy; DHRD, Doyne honeycomb retinal dystrophy; FAC, Ferric ammonium citrate; RDM, Retinal differentiation media.

\section{Publisher's note}

Springer Nature remains neutral with regard to jurisdictional claims in published maps and institutional affiliations.

The online version of this article (https://doi.org/10.1038/s41420-019-0171-9) contains supplementary material, which is available to authorized users.
Received: 5 March 2019 Revised: 19 March 2019 Accepted: 21 March 2019 Published online: 16 May 2019

\section{References}

1. Seddon, J. M., Cote, J., Page, W. F., Aggen, S. H. \& Neale, M. C. The US twin study of age-related macular degeneration: relative roles of genetic and environmental influences. Arch. Ophthalmol. 123, 321-327 (2005).

2. Velilla, S. et al. Smoking and age-related macular degeneration: review and update. J. Ophthalmol. 2013, 895147 (2013).

3. Klein, R., Knudtson, M. D., Cruickshanks, K. J. \& Klein, B. E. Further observations on the association between smoking and the long-term incidence and progression of age-related macular degeneration: the Beaver Dam Eye Study. Arch. Ophthalmol. 126, 115-121 (2008).

4. Fujihara, M., Nagai, N., Sussan, T. E., Biswal, S. \& Handa, J. T. Chronic cigarette smoke causes oxidative damage and apoptosis to retinal pigmented epithelial cells in mice. PLoS ONE 3, e3119 (2008).

5. Bertram, K. M., Baglole, C. J., Phipps, R. P. \& Libby, R. T. Molecular regulation of cigarette smoke induced-oxidative stress in human retinal pigment epithelial cells: implications for age-related macular degeneration. Am. J. Physiol. Cell Physiol. 297, C1200-C1210 (2009).

6. Chen, H., Lukas, T. J., Du, N., Suyeoka, G. \& Neufeld, A. H. Dysfunction of the retinal pigment epithelium with age: increased iron decreases phagocytosis and Iysosomal activity. Investig. Ophthalmol. Vis. Sci. 50, 1895-1902 (2009).

7. He, X. et al. Iron homeostasis and toxicity in retinal degeneration. Prog. Retin. Eye Res. 26, 649-673 (2007).

8. Wong, R. W., Richa, D. C., Hahn, P., Green, W. R. \& Dunaief, J. L. Iron toxicity as a potential factor in AMD. Retina 27, 997-1003 (2007).

9. Hahn, P., Ying, G. S., Beard, J. \& Dunaief, J. L. Iron levels in human retina: sex difference and increase with age. Neuroreport 17, 1803-1806 (2006).

10. Chen, $\mathrm{H}$. et al. Changes in iron-regulatory proteins in the aged rodent neural retina. Neurobiol. Aging 30, 1865-1876 (2009).

11. Hahn, P., Milam, A. H. \& Dunaief, J. L. Maculas affected by age-related macular degeneration contain increased chelatable iron in the retinal pigment epithelium and Bruch's membrane. Arch. Ophthalmol. 121, 1099-1105 (2003).

12. Dunaief, J. L. et al. Macular degeneration in a patient with aceruloplasminemia, a disease associated with retinal iron overload. Ophthalmology 112, 1062-1065 (2005)

13. Yamaguchi, K., Takahashi, S., Kawanami, T., Kato, T. \& Sasaki, H. Retinal degeneration in hereditary ceruloplasmin deficiency. Ophthalmologica 212, 11-14 (1998).

14. Gnana-Prakasam, J. P. et al. Iron-mediated retinal degeneration in haemojuvelin-knockout mice. Biochem. J. 441, 599-608 (2012).

15. Gnana-Prakasam, J. P. et al. Absence of iron-regulatory protein Hfe results in hyperproliferation of retinal pigment epithelium: role of cystine/glutamate exchanger. Biochem. J. 424, 243-252 (2009).

16. Hahn, P. et al. Disruption of ceruloplasmin and hephaestin in mice causes retinal iron overload and retinal degeneration with features of age-related macular degeneration. Proc. Natl Acad. Sci. USA 101, 13850-13855 (2004).

17. Wong, W. L. et al. Global prevalence of age-related macular degeneration and disease burden projection for 2020 and 2040: a systematic review and metaanalysis. Lancet Glob. Health 2, e106-e116 (2014).

18. Clemons, T. E. et al. Risk factors for the incidence of advanced age-related macular degeneration in the age-related eye disease study (AREDS) AREDS report no. 19. Ophthalmology 112, 533-539 (2005).

19. Jamal, A. et al. Current cigarette smoking among adults-United States, 2016. Morb. Mortal. Wkly. Rep. 67, 53-59 (2018).

20. Sharma, A. et al. Effects of benzo(e)pyrene, a toxic component of cigarette smoke, on human retinal pigment epithelial cells in vitro. Investig. Ophthalmol. Vis. Sci. 49, 5111-5117 (2008).

21. Jia, L. et al. Acrolein, a toxicant in cigarette smoke, causes oxidative damage and mitochondrial dysfunction in RPE cells: protection by (R)-alpha-lipoic acid. Investig. Ophthalmol. Vis. Sci. 48, 339-348 (2007).

22. Sundelin, S., Wihlmark, U., Nilsson, S. E. \& Brunk, U. T. Lipofuscin accumulation in cultured retinal pigment epithelial cells reduces their phagocytic capacity. Curr. Eye Res. 17, 851-857 (1998).

23. Zhou, J., Kim, S. R., Westlund, B. S. \& Sparrow, J. R. Complement activation by bisretinoid constituents of RPE lipofuscin. Investig. Ophthalmol. Vis. Sci. 50, 1392-1399 (2009) 
24. Saadat, K. A. et al. Inhibition of autophagy induces retinal pigment epithelial cell damage by the lipofuscin fluorophore A2E. FEBS Open Bio. 4, 1007-1014 (2014).

25. Feeney-Burns, L., Hilderbrand, E. S. \& Eldridge, S. Aging human RPE: morphometric analysis of macular, equatorial, and peripheral cells. Investig. Ophthalmol. Vis. Sci. 25, 195-200 (1984)

26. Wolkow, N. et al. Aceruloplasminemia: retinal histopathologic manifestations and iron-mediated melanosome degradation. Arch. Ophthalmol. 129, 1466-1474 (2011).

27. Kurz, T., Terman, A., Gustafsson, B. \& Brunk, U. T. Lysosomes in iron metabolism, ageing and apoptosis. Histochem. Cell Biol. 129, 389-406 (2008).

28. Brunk, U. T. \& Terman, A. Lipofuscin: mechanisms of age-related accumulation and influence on cell function. Free Radic. Biol. Med. 33, 611-619 (2002).

29. Regan, C. M., de Grip, W. J., Daemen, F. J. \& Bonting, S. L. Degradation of rhodopsin by a lysosomal fraction of retinal pigment epithelium: biochemical aspects of the visual process. XLI. Exp. Eye Res. 30, 183-191 (1980).

30. Rakoczy, P. E. et al. Modulation of cathepsin D activity in retinal pigment epithelial cells. Biochem. J. 324, 935-940 (1997). (Pt 3).

31. Monick, M. M. et al. Identification of an autophagy defect in smokers' alveolar macrophages. J. Immunol. 185, 5425-5435 (2010).

32. Wang, A. L. et al. Changes in retinal pigment epithelium related to cigarette smoke: possible relevance to smoking as a risk factor for age-related macular degeneration. PLOS ONE 4, e5304 (2009).

33. Saini, J. S. et al. Nicotinamide ameliorates disease phenotypes in a human PSC model of age-related macular degeneration. Cell Stem Cell 20, 635-647 (2017).

34. Galloway, C. A. Drusen in patient-derived hiPSC-RPE models of macular dystrophies. Proc. Natl Acad.Sci. USA, E8214-E8223 (2017).

35. Hallam, D. et al. An induced pluripotent stem cell patient specific model of complement factor $\mathrm{H}(\mathrm{Y} 402 \mathrm{H})$ polymorphism displays characteristic features of age-related macular degeneration and indicates a beneficial role for UV light exposure. Stem Cells 35, 2305-2320 (2017).

36. Singh, R. et al. iPS cell modeling of best disease: insights into the pathophysiology of an inherited macular degeneration. Hum. Mol. Genet. 22, 593-607 (2013).

37. Marmorstein, A. D. et al. Mutant best1 expression and impaired phagocytosis in an iPSC model of autosomal recessive bestrophinopathy. Sci. Rep. 8, 4487 (2018).

38. Lukovic, D. et al. Human iPSC derived disease model of MERTK-associated retinitis pigmentosa. Sci. Rep. 5, 12910 (2015).

39. Ramsden, C. M. et al. Rescue of the MERTK phagocytic defect in a human iPSC disease model using translational read-through inducing drugs. Sci. Rep. 7, 51 (2017).

40. Sonoda, S. et al. A protocol for the culture and differentiation of highly polarized human retinal pigment epithelial cells. Nat. Protoc. 4, 662-673 (2009).

41. Barisani, D., Meneveri, R., Ginelli, E., Cassani, C. \& Conte, D. Iron overload and gene expression in HepG2 cells: analysis by differential display. FEBS Lett. 469 208-212 (2000)

42. Gnana-Prakasam, J. P. et al. Expression and iron-dependent regulation of succinate receptor GPR91 in retinal pigment epithelium. Investig. Ophthalmol. Vis. Sci. 52, 3751-3758 (2011).

43. Voloboueva, L. A., Killilea, D. W., Atamna, H. \& Ames, B. N. N-tert-butyl hydroxylamine, a mitochondrial antioxidant, protects human retinal pigment epithelial cells from iron overload: relevance to macular degeneration. FASEB J. 21, 4077-4086 (2007).

44. Wolkow, N. et al. Iron upregulates melanogenesis in cultured retinal pigment epithelial cells. Exp. Eye Res. 128, 92-101 (2014).

45. Murray, A. R., Fliesler, S. J. \& Al-Ubaidi, M. R. Rhodopsin: the functional significance of asn-linked glycosylation and other post-translational modifications. Ophthalmic Genet. 30, 109-120 (2009).

46. Lemaitre, V., Dabo, A. J. \& D'Armiento, J. Cigarette smoke components induce matrix metalloproteinase-1 in aortic endothelial cells through inhibition of mTOR signaling. Toxicol. Sci. 123, 542-549 (2011).

47. Cano, M. et al. Cigarette smoking, oxidative stress, the anti-oxidant response through Nrf2 signaling, and age-related macular degeneration. Vis. Res. 50 652-664 (2010).

48. Geissler, C. \& Singh, M. Iron, meat and health. Nutrients 3, 283-316 (2011).

49. Hayasaka, S., Hara, S. \& Mizuno, K. Degradation of rod outer segment proteins by cathepsin D. J. Biochem. 78, 1365-1367 (1975).

50. Sparrow, J. R. \& Duncker, T. Fundus autofluorescence and RPE lipofuscin in age-related macular degeneration. J. Clin. Med. 3, 1302-1321 (2014).
51. Marmorstein, A. D., Marmorstein, L. Y., Sakaguchi, H. \& Hollyfield, J. G. Spectral profiling of autofluorescence associated with lipofuscin, Bruch's membrane, and sub-RPE deposits in normal and AMD eyes. Investig. Ophthalmol. Vis. Sci. 43, 2435-2441 (2002).

52. Bhutto, I. \& Lutty, G. Understanding age-related macular degeneration (AMD): relationships between the photoreceptor/retinal pigment epithelium/Bruch's membrane/choriocapillaris complex. Mol. Asp. Med. 33, 295-317 (2012).

53. Bonilha, V. L. Age and disease-related structural changes in the retinal pigment epithelium. Clin. Ophthalmol. 2, 413-424 (2008).

54. Sparrow, J. R., Hicks, D. \& Hamel, C. P. The retinal pigment epithelium in health and disease. Curr. Mol. Med. 10, 802-823 (2010).

55. Keeling E., Lotery A. J., Tumbarello D. A., \& Ratnayaka J. A. Impaired cargo clearance in the retinal pigment epithelium (RPE) underlies irreversible blinding diseases. Cells 7 (2018), https://doi.org/10.3390/cells7020016.

56. Lin, B. et al. Clinical and genetic analyses reveal novel pathogenic ABCA4 mutations in Stargardt disease families. Sci. Rep. 6, 35414 (2016).

57. Esteve-Rudd, J. et al. Defective phagosome motility and degradation in cell nonautonomous RPE pathogenesis of a dominant macular degeneration. Proc. Natl Acad. Sci. USA 115, 5468-5473 (2018).

58. Singh, R. et al. Pharmacological modulation of photoreceptor outer segment degradation in a human iPS cell model of inherited macular degeneration. Mol. Ther. 23, 1700-1711 (2015).

59. Bindewald, A. et al. Classification of fundus autofluorescence patterns in early age-related macular disease. Investig. Ophthalmol. Vis. Sci. 46, 3309-3314 (2005).

60. Lueck, K., Hennig, M., Lommatzsch, A., Pauleikhoff, D. \& Wasmuth, S. Complement and UV-irradiated photoreceptor outer segments increase the cytokine secretion by retinal pigment epithelial cells. Investig. Ophthalmol. Vis. Sci. 53, 1406-1413 (2012).

61. Hadziahmetovic, M. et al. Age-dependent retinal iron accumulation and degeneration in hepcidin knockout mice. Investig. Ophthalmol. Vis. Sci. 52, 109-118 (2011).

62. Sparrow, J. R. \& Boulton, M. RPE lipofuscin and its role in retinal pathobiology. Exp. Eye Res. 80, 595-606 (2005).

63. Kinnunen, K., Petrovski, G., Moe, M. C., Berta, A. \& Kaarniranta, K. Molecular mechanisms of retinal pigment epithelium damage and development of agerelated macular degeneration. Acta Ophthalmol. 90, 299-309 (2012).

64. Ueda, K. et al. Iron promotes oxidative cell death caused by bisretinoids of retina. Proc. Natl Acad. Sci. USA 115, 4963-4968 (2018).

65. Hernández, D. et al. Electrical stimulation promotes cardiac differentiation of human induced pluripotent stem cells. Stem Cells Int. 2016, 1718041 (2016).

66. Phillips, M. J. et al. Blood-derived human iPS cells generate optic vesicle-like structures with the capacity to form retinal laminae and develop synapses. Investig. Ophthalmol. Vis. Sci. 53, 2007-2019 (2012).

67. Galloway, C. A. et al. Characterization of human iPSC-RPE on a prosthetic Bruch's membrane manufactured from silk fibroin. Investig. Ophthalmol. Vis. Sci. 59, 2792-2800 (2018).

68. Singh, R. et al. Functional analysis of serially expanded human iPS cell-derived RPE cultures. Investig. Ophthalmol. Vis. Sci. 54, 6767-6778 (2013).

69. Meyer, J. S. et al. Modeling early retinal development with human embryonic and induced pluripotent stem cells. Proc. Natl Acad. Sci. USA 106, 16698-16703 (2009).

70. Meyer, J. S. et al. Optic vesicle-like structures derived from human pluripotent stem cells facilitate a customized approach to retinal disease treatment. Stem Cells 29, 1206-1218 (2011).

71. Baglole, C. J. et al. The aryl hydrocarbon receptor attenuates tobacco smokeinduced cyclooxygenase-2 and prostaglandin production in lung fibroblasts through regulation of the NF-kappaB family member RelB. J. Biol. Chem. 283, 28944-28957 (2008).

72. Martey, C. A., Baglole, C. J., Gasiewicz, T. A., Sime, P. J. \& Phipps, R. P. The aryl hydrocarbon receptor is a regulator of cigarette smoke induction of the cyclooxygenase and prostaglandin pathways in human lung fibroblasts. Am. J. Physiol. Lung Cell Mol. Physiol. 289, L391-L399 (2005).

73. Singh, R. Functional analysis of serially expanded human iPS cell-derived RPE cultures. Investig. Ophthalmol. Vis. Sci 54, 6767-6778 (2013).

74. Gogly, B., Groult, N., Hornebeck, W., Godeau, G. \& Pellat, B. Collagen zymography as a sensitive and specific technique for the determination of subpicogram levels of interstitial collagenase. Anal. Biochem. 255 211-216 (1998). 